\title{
Decision Making for Predictive Maintenance in Asset Information Management
}

\author{
R. B. Faiz and Eran A. Edirisinghe \\ Department of Computer Science, \\ Loughborough University, Leicestershire, UK
}

\author{
R.faiz@lboro.ac.uk; e.a.edirisinghe@lboro.ac.uk
}

\begin{abstract}
Asset management is a process of identification, design, construction, operation, and maintenance of physical assets (Wenzler, 2005). An asset-centric approach is vital for the success of an asset intensive organisation as the effective management of assets is a major determinant of organisational success. One key issue in asset information management is the availability of information at the right time, in the right format, before the right person, against the right query, and at the right level. This paper provides a comprehensive and in-depth critical analysis from literature which fulfils an identified need of fusing asset information for predictive maintenance so that decision making can be improved. The critical literature review included also highlights the need for an expert system which integrates reliable information with effective decision-support, under the umbrella of Asset Management. Various elements of asset management were critically reviewed, highlighting the need for more robust Predictive maintenance management for assets. We argue that this is best achieved by a system that, in particular, incorporates Expert System to enhance the quality of predictive maintenance through accurate decision analysis. In addition, it should have fuzzy logic reasoning ability that assists in the decision-making process. Our analysis leads us to propose that Expert System when combined with fuzzy logic provides a better way of decision making in predictive maintenance management of assets.
\end{abstract}

Keywords: Asset management, predictive maintenance, expert system, fuzzy logic, decision making.

\section{Introduction}

All organizations with assets such as processing equipment, servers, railway tracks, or pipelines share a common problem: how can they be kept operational for as long as possible and as economically as possible without sacrificing reliability or safety?

The answer, of course, is appropriate maintenance. These problems can be mitigated by taking a systematic approach to active management of assets. The first requirement is accurate and up to

Material published as part of this publication, either on-line or in print, is copyrighted by the Informing Science Institute. Permission to make digital or paper copy of part or all of these works for personal or classroom use is granted without fee provided that the copies are not made or distributed for profit or commercial advantage AND that copies 1) bear this notice in full and 2) give the full citation on the first page. It is permissible to abstract these works so long as credit is given. To copy in all other cases or to republish or to post on a server or to redistribute to lists requires specific permission and payment of a fee. Contact Publisher@InformingScience.org to request redistribution permission. date information about the assets. From this, predictions can then be made and acted on.

A well-implemented integrated asset management and maintenance system can impact every part of an organization, increasing asset uptimes, reducing maintenance costs, increasing profits, and enhancing the reputation of the business with its customers. When reli- 
able information and effective decision-support tools are integrated under an asset management umbrella, the costs for maintenance, repair, and renewal are substantially reduced.

Availability of asset information before the asset manager at the right time and against the right query has been a fundamental challenge in past. This can be answered by incorporating effective decision analysis on asset information for better predictive maintenance management. An asset manager has a knowledge base of asset information; even then, when it comes to predictive maintenance, the asset manager may not be well equipped to perform better decision analysis for critical decision making that results in effective maintenance.

This paper presents a methodology which is based on fusing an Expert System with a fuzzy logic scheme to provide a better way of decision making with predictive maintenance in Predictive Maintenance of Asset Management regime. Thus its focus is on decision making in asset information management for better predictive maintenance.

In the first section of this paper an introduction of an asset management system is explained. The second section reviews literature of current asset information management systems, decision making, and predictive maintenance in practice. In the third section asset management, decision making, and predictive maintenance are critically evaluated. Based on this critical evaluation, in the fourth section an effective and more robust asset information management system is proposed that incorporates better decision making ability for reliable predictive maintenance. The fifth and final section concludes that high-quality decisions cannot be expected without high-quality information.

\section{Asset Management}

Asset management is a process of identification, design, construction, operation, and maintenance of physical assets. Over the years a number of different definitions and approaches to asset management have been presented. A popular definition is that "asset management is concerned with obtaining and using the knowledge needed to optimize trade-offs among financial performance, and operational performance" (Ness \& Lambie, 2004). Further, the science of asset management aims to equip engineers to become businessmen and introduces structured methods for handling reliability, performance, and maintenance (Woodhouse, 2001). An individual who is responsible in managing organizational assets, for example, an asset manager, is considered a pivot that bridges the gap between business objectives and the considerable complexities of technical and human issues. An Asset Manager acts as a professional translator by converting options, such as asset design or maintenance strategies or asset replacement decisions, into business language.

A typical asset management system observes assets for signs of failure or degradation and schedules maintenance intervention before critical failure occurs. This information is also used to maintain up to date centralized and accurate information on the nature, location, and condition of all the assets across the business.

This alone results in advantages such as the ability to minimize unforeseen disruptions to operations. Increasing confidence in the system maximizes the overall uptime of individual assets and reductions of serious problems (which require time-intensive repair). Better information on the condition of assets, and their degradation characteristics, allows maintenance regimes to be streamlined. This enables optimum use of time and resources so that assets are kept in better shape and there are fewer maintenance surprises. Further, accurate and comprehensive information on asset condition means that early warnings can be given of possible safety-critical problems, thus preventing accidents (Clarke, 2005).

Strategic Asset Management Systems, specifically, help in maintaining assets throughout their useful life span. Besides they support the asset manager in managing assets for the benefit of the 
organization as a whole. Such systems maximize the performance of fixed, physical, or capital assets rather than financial assets or intangible assets such as knowledge capital that have a direct and significant impact on achieving organizational objectives. The question of how to classify assets as strategic is best answered by the organization itself. However, managing critical assets to optimize their value is universally important. The essence of asset management is integration, synchronization, and optimization of asset management practices across multiple types of assets, across the entire enterprise at a strategic and tactical level (MRO, 2004a).

No asset management system can eliminate all failures. An asset monitoring system can only cope with problems that it has been designed or trained to recognize. The process usually starts with information collection and analysis. This helps in understanding behaviour of the assets. The next step is to develop sufficient reliability decision support models and refine the models in the light of how they perform maintenance activities automatically until the system is sufficiently mature (Clarke, 2005).

An asset management system is diversified and can have various components with great variations in their functionality. For example, different assets will be important for different companies thus requiring different asset management systems.

Any methodology implemented for asset management in an organization may provide a framework for collecting the information required to make decisions about the strategic plans of an organization (Vanier, 2001).

This paper specifically focuses on decision making in asset information management for better predictive maintenance of assets. Therefore, among all the components of an asset information management system, it emphasizes primarily decision making and predictive maintenance.

\section{Decision Making}

As decision making involves many options, computer-based decision support systems are developed to assist decision makers in considering the implications of various courses of action and can help reduce the risk of human errors.

A Decision Support System (DSS) is a tool used to improve the process of decision making in complex systems, particularly where information is uncertain or incomplete.

DSS are used extensively in business and industry to assist in decision-making across a wide spectrum of problem areas. There are a number of approaches and techniques employed, from simple information reporting tools, to sophisticated Artificial Intelligence systems using Bayesian statistics or genetic algorithms (Rippen, 2005). They all share three common components of decision support systems:

a. An information store house of knowledge.

b. A process by which this knowledge may be systematically interrogated to provide answers to questions. This is the component that predominantly distinguishes between different Decision Support Systems.

c. A user interface providing users with a perceptive, accessible tool for gaining the information they require.

Decision making can be classified in to two broad categories; it can be either a manual or an automated decision. 


\section{Manual decision making}

Decisions can be made with the help of Graphical Tools or Diagrammatic tools like graphs, flow charts, and Entity-relationship diagrams. Let us take an example of a common scenario where one person is responsible for manual checking using Entity Relationship Diagrams (ERD) made by students of the same class for a given problem. As all students are addressing one problem so many of them will identify the same entities. Thus, the marker will check similar components in different diagrams manually and repeatedly. This will result in redundant checking of entities in different diagrams as the checker has to go through similar entities again and again.

This constraint can be minimized by automated checking and thus all similar entities can be found and marked out instead of repeated checking. This clearly reflects the importance of automation, or, in other words, intelligent software is vital for repeated processing.

\section{Knowledge based system / expert system}

A knowledge-based system is a program for querying a knowledge base. It provides the means for the computerized collection, organization, and retrieval of knowledge.

Expert systems are generally designed to be experts in one problem domain. The advantage of applying expert systems to assist problem solving is that the confidence in correct decisions can be greatly increased (Clarke, 2005). In particular:

a. There are various expert systems in which a "rule base" and an "inference engine" cooperate to simulate the reasoning process that a human expert uses in analyzing a problem and arriving at conclusions.

b. They provide consistent answers for repetitive decisions and tasks.

c. Significant levels of information can be maintained.

d. Many transactions that human experts may overlook can be reviewed.

Case based reasoning (CBR): CBR stores a set of problems and answers in an organized data structure called a Case-Base or Case Archive. A Case Based Reasoning system, upon being presented with a problem, finds the case in its knowledge base that is most closely related to the new problem and presents that case's solution as an output, with suitable modifications.

Much of the power of decision analysis lies in its ability to effectively integrate the many factors that commonly affect a decision. Such an integrating capacity makes decision analysis a very useful means of facilitating the decision-making process. CBR can help decision-makers identify what features of a problem are the important ones to remember during problem solving.

CBR has many advantages for analyzing a class of decisions, they are:

a. Allows decision-makers to propose solutions to problems quickly without the need to derive those solutions from scratch. This provides organizational memory-based intuition for a given problem, to avoid any irregular or abnormal problem solving process.

b. Provide a systematic mechanism for storing domain-dependent knowledge and decisionanalytic knowledge as cases, and reuse them according to the characteristics of problems.

c. Based on past mistakes made by some decision makers in an organization, CBR can alert decision makers to avoid repeating past mistakes (Lee \& Kim, 2002).

Other advantages are that it includes a system that can learn incrementally and has explanation capability. For example, even though one of the prevalent adaptive decision-making methods, knowledge bases, has received a lot of attention because of its high predictability, its lack of ex- 
planation capability prevents usage in many areas. CBR is relatively easy to understand in terms of how the results are produced and which cases are used, appropriate method for many realworld areas, in particular those that need explanation.

CBR can be effective even if the knowledge base or domain theory is incomplete. Certain techniques of automated learning, such as explanation- based learning, work well when only a strong domain theory exists, whereas CBR can use many examples to overcome the gaps in a weak domain theory while still taking advantage of the domain theory. These characteristics of CBR make it appropriate for diagnosis, prognosis, and prescription in medicine, since medical domains put more stress on real cases than other domains. In addition, the explanation capability is essential for these areas.

A further advantage of CBR is the relative ease of combining it with other approaches. For example, CBR has been combined with knowledge bases in a diagnosis system and together with a rule-based system for diagnosis of heart failure (Park, Kim, \& Chun, 2006).

Fuzzy logic: Fuzzy logic is not any less precise than any other form of logic: it is an organized and mathematical method of handling inherently imprecise concepts. To support the above argument one could refer to the following example:

The concept of coldness cannot be expressed in a mathematical equation, as although temperature is a quantity, coldness is not. However, people have an idea of what cold is and agree that there is no sharp cutoff between cold and not cold, where something is cold at X degrees but not cold at X+1 degrees (Negnevitsky, 2002).

The description of a conflict situation and the formulation of expert knowledge regarding appropriate actions for this conflict are frequently vague. Fuzzy concepts have emerged as a suitable means to deal effectively with vague concepts.

Here follows a typical scenario that serves as an example of a functioning system: in an airport flight schedule aeroplane $\mathrm{Y}$ waited for aeroplane $\mathrm{X}$ to transfer passengers from aeroplane $\mathrm{X}$ to aeroplane $\mathrm{Y}$. Since aeroplane $\mathrm{X}$ was late, a decision had to be made whether aeroplane $\mathrm{Y}$ should wait or depart. The concern of the transfer passengers of aeroplane $X$ have to be balanced against those of passengers of aeroplane $\mathrm{Y}$ who intend to board aeroplane $\mathrm{Y}$ and hence an optimum airport flight schedule decision has to be made regarding the divergent aims mentioned above.

The decision is influenced by the following conditions:

a. Length of the delay of aeroplane X,

b. The number of transfer passengers,

c. The length of the further trip of aeroplane $\mathrm{Y}$,

d. The time interval of aeroplane in the direction of aeroplane $\mathrm{Y}$ and whether aeroplane $\mathrm{Y}$ is the last flight in this direction on this day (and, thus, the last chance for transfer passengers to reach their destination on that particular day) (Fay, 2002).

\section{Predictive Maintenance (PM)}

PM is another vital component of asset management systems. Maintenance can be either corrective fixing of failure or it can be preventive, that is, it predicts the failure before it happens.

Through predictive maintenance we can prevent components or systems from malfunctioning and ensure the intended functions can be carried out throughout their service life (Kyle, Vanier, Kosovac, \& Froese, 2000).

PM avoids excessive cost normally incurred by corrective maintenance. 
The distinctive characteristics of PM are:

a. Mitigates failure;

b. Finds the commencement of failure;

c. Uncovers hidden failure.

There are two key approaches to PM.

\section{Time directed (TD)}

Time Directed tasks are performed to prevent or retard failures and are carried out at fixed time intervals regardless of the availability of other information that occurs during the preset time. A TD task also requires an interruption into the equipment, thereby rendering it out of service until the task is completed.

\section{Condition based maintenance (CBM)}

$\mathrm{CBM}$ is also known as on-condition maintenance, condition-directed maintenance, or predictive maintenance. It is designed to detect failure, which is an appropriate option for PM when the following conditions apply:

a. Either failure prevention is not feasible, or how it can be achieved is not yet known, as in cases where the event leading to failure occurs in a predominantly random manner;

b. A measurable parameter which correlates with the onset of failure has been identified, for example, the solid content in the lubricant is an indicator of the machine's wearing condition;

c. It is possible to identify a value of that parameter when action may be taken before full failure occurs, such as the setting of warning limits for the solids content of the lubricant.

A major class of decision problems in CBM relates to the issue of inspection. It deals with situations in which inspection tasks are performed at periodic intervals to determine the true state of the equipment. Based on the result of each inspection, two decisions will be made:

a. What maintenance action to take? To replace or repair the system to a specific state or to leave it as is;

b. When is the next inspection?

There is a widespread belief that corrective maintenance is always less economical than preventive maintenance, and all failures can be prevented. As a result, time-directed maintenance becomes the norm of preventive maintenance action, motivating the indiscriminate use of overhaul or preventive replacement procedures in PM programmes. This approach to PM firstly wastes a lot of resources in doing unnecessary tasks which will not improve equipment or system availability, and secondly it is potentially risky (Tsang, 1995).

A principal argument in favour of detailed and integrated asset information management system is that accurate and unchallenged information is available to anyone with the skills to analyse and interpret it for the benefit of a company (Sherwin, 2000).

Predictive scheduling relies more on information and explicit knowledge and, in contrast, reactive scheduling decisions are more tacit.

There are five major types of predictive and reactive scheduling approaches. For ease of understanding we present them in the context of a rail network asset management system. 
a. Spatial: The predictive scheduling approach considers the network wide impacts of the scheduling decisions, while the reactive approach focuses on local impacts due to lack of network wide information and knowledge required for fast decision making.

b. Chronological: A predictive schedule is developed for a few days or weeks and can be used repeatedly, unlike a reactive schedule that is created for the next few hours and is not reusable.

c. Comprehensiveness: The predictive schedule covers the entire journeys of all trains; whereas, the reactive schedule is a partial schedule which covers trains that travel within a region, i.e., the territory under the control of the regional train dispatchers.

d. Decision Level: The main outlook of predictive schedule generation is planning the appropriate and balanced use of railway assets, primarily, according to the economic and social objectives of the company; whereas, the reactive schedules are generated to tackle the consequences of day-to-day operational problems.

e. Management Level: A predictive schedule is used to coordinate train operations across different geographical regions in advance, while the reactive schedule coordinates stations within the region to comply with the master schedule as far as possible (Isaai \& Cassaigne, 2001).

\section{Future Directions}

The last few years have seen a rapid growth of interest in combining expert system techniques and fuzzy logic as a new paradigm Do you have recent citations you can add here (Fu, 1995). This hybrid idea is largely a consequence of an increasingly strong belief that expert systems and fuzzy logic can complement each other beneficially.

The integration of expert systems and fuzzy logic into a system can be explored in terms of their useful relationships in the system (Fu, 1995).

\section{Asset Information Management}

If a company really knew what they should know they will be unbeaten. Better and more efficient information management is the key to better decision-making (Vanier, 2001). Some prominent areas that offer resistance in Asset Information Management include lack of knowledge by the whole asset information management team especially in higher level management and also obsolete information provided by old systems. Other important areas are:

\section{Information collection}

Asset information collection requires the user to address the following considerations:

a. What information is needed and of what quality?

b. What information is available?

c. What are information cleansing priorities?

d. Which tools facilitate visibility, access, and integration of information?

e. How to sustain the quality of cleansed information?

f. How to measure the effectiveness of asset information management? (Network Rails, 2005).

\section{Overloading of data}

Too much data and not enough information can lead to disastrous mismanagement. Besides it can give rise to other misrepresentations and controversies (Institute of Asset Management [IAM], 2003). 


\section{Ambiguous organizational objectives}

Organizations tend to collect information that is easiest to collect, irrespective of the need for it or the subsequent usefulness. The setting of departmental objectives is also based on such thinking; maintainers and technical service providers may be given budget targets or deadlines irrespective of the potential 'trade-off' impact against operational performance. Production, operations, or customer relations personnel, on the other hand, are motivated and measured in the terms of output volumes or quality, irrespective of the costs incurred by others to achieve such output. Hence the current scenario requires an asset management system which connects to organizational objectives (IAM, 2003).

\section{Weak correlation between apparent indicators and the risks}

In some areas the volume of information is too low to be able to scrutinize safety performance. For example, there are too few real accidents and fatalities to be used as the apparent indicators, and we can not wait to see how many people are killed before we do something to improve the situation. Near misses and minor incidents are measured, giving enough statistical material to view trends. From these and some other assumptions, we can conclude the underlying risks of fatal events. However the ratio is often weak and the reporting of lesser events can easily become distorted by fear of accountability. The symptoms that are available to be monitored may only loosely indicate the underlying risks or performance. Therefore it is important for any predictive asset management system to overcome these limitations (IAM, 2003).

\section{Trade off between cost and risk}

Cost and risk trade off, in particular, is difficult to handle as there is no convenient existing feature that takes account of risk exposure and costs in a unified approach. The search is ongoing for a rational business measure that combines risks and costs into such a performance indicator (IAM, 2003).

Asset Management is described as finding the best compromise and convincing the various stakeholders that such a compromise is indeed optimal (Woodhouse, 2000).

Finding a true optimum is difficult because there are two specific barriers in identifying where the optimum lies:

a. Insufficient and lack of quality of relevant information: It refers to major risks and how would they vary with more/less preventive action.

b. Making use of available information: One of the common myths in terms of information manipulation is if we do not have appropriate information, how can we improve its usage mechanisms? The traditional reaction to poor information and subjective decision-making is to start collecting more/better information and hope that it will somehow assist us in what to do.

Without knowing how the information can be used, it is not possible to know what information is worth collecting. Even if it was possible to guess correctly the information that is needed, how (and when) would one know that sufficient information has been collected? What is "sufficient," and is it physically/economically possible to collect it? Without a clear idea of how it will be used, and sensitivity to information inaccuracy, it is impossible to say what information is needed and to what precision.

The first challenge is, therefore, the understanding of what information is required for specific decisions, and how it should be used. This issue can be addressed by making sure that the right questions are asked in the first place. Broadly speaking asset management can be divided into two main decision making categories: 
a. Decisions regarding direction: Where are we going and how can we get there?

b. Decisions regarding structure: How do we organise and what has to be done?

As both are prime contributors in asset management, attention to the latter without addressing the former can result in "doing the wrong work faster" which does not guarantee better overall performance (Woodhouse, 2001).

\section{Predictive Maintenance}

An asset manager has a knowledge base of asset information; even then, when it comes to predictive maintenance, an asset manager may not be well equipped to perform decision analysis for critical decision making that results in effective predictive maintenance. Thus he has to be guided by predictive maintenance. Again for ease of understanding we present them related to a rail network asset management system.

\section{Predictive scheduling}

The timetable planners who develop predictive schedules manually assume that the resources required for implementing the schedule in the real world are all available. Furthermore, they usually overlook the uncertain accuracy of information (e.g., running time between adjacent stations) and use information confidently. They also rely to a notable extent on the information and knowledge used in previous timetable production to produce reasonable and acceptable schedules. It has been shown in literature that decision technologies can improve scheduling decisions under uncertainty, for instance, enabling the schedulers to study the reliability of created schedules via allowing certain parameters (e.g., running time) to vary randomly (Isaai \& Cassaigne, 2001).

\section{Reactive scheduling}

In contrast with predictive scheduling decisions, reactive scheduling decisions are made when the current schedule is disrupted or additional trains and operations are scheduled. It is often very difficult to predict all consequences of the new scheduling decisions, and, thus, expert judgments are made on the basis of imperfect information and previous experiences with a relatively shortterm view of the future impacts of decisions. As the decisions are to be executed shortly after they are made, communication with on-board and station crews and adjoining regions' schedulers is an essential part of the work.

\section{Future Direction in Decision Making}

Complete automation is of course not possible unless perfect information is analysed and processed through a perfect model. Managerial decisions are normally based on human judgement (i.e., guesswork informed by experience and supplemented by partial calculations based on information and knowledge). It is not so much that the decisions themselves must become automatic as that they must be taken more quickly by fewer managers who must therefore have better information provided by a more complex and integrated asset management system, capable of suggesting answers by calculating the results of self-generated alternatives. As a computer cannot make judgements but only calculations, the input information must become more detailed, so that the modelling can be more accurate and the answers sufficiently precise to choose rationally between alternative policies (Sherwin, 2000).

A decision support system will not eliminate 'bad' decisions or badly drawn conclusions derived from asking the 'wrong' questions. The output of the DSS must be examined critically and used together with existing understanding of the wider business or application areas. 
The increasing level of decision support system implementation in organizations over past two decades is a strong evidence to show that DSS is a viable and well accepted managerial tool (Eom, Lee, Kim, \& Somarajan, 1998). This being said, systems are now being developed that are providing enormous benefits, both in time and cost savings (Rippen, 2005; Weidl, Madsen, \& Dahlquist, 2002).

DSS assist in evaluating different maintenance decisions in order to select the most robust and cost-effective solution in a systematic and transparent way (Zoeteman, 2001).

Currently available decision models are commonly designed to optimize a single criterion, such as minimizing the total maintenance related costs or maximizing equipment availability. Since decision making in practice is often characterized by the need to satisfy multiple goals, the formulation of multi-criteria decision models is another worthwhile topic of future research work in inspection problems (Tsang, 1995).

An effective decision-support system is needed by asset managers to assist them in identifying optimal maintenance strategies that minimize life-cycle costs and risk of failure (Lounis, Vanier, \& Lacasse, 1999).

Therefore a fundamental research issue in building an intelligent decision support system involves linking the domain-specific knowledge of experts with the normative power of decision analysis to improve the quality of decisions (Yam, Tse, Li, \& Tu, 2001).

\section{Future directions in knowledge base systems / expert systems}

The relevant literature clearly reveals several future research areas in the domain of expert systems, some of which are highlighted below.

Some key areas to address in the future to improve the performance of current expert systems:

a. Inclusion of human-like common sense needed in some decision making

b. The inclusion of creative responses: human experts can respond innovatively in unusual circumstances

c. Domain experts not always being able to explain their logic and reasoning

d. The challenges of automating complex processes

e. The lack of flexibility and ability to adapt to changing environments

f. Not being able to respond appropriately when no answer is available (Negnevitsky, 2002).

One of the major problems with expert reasoning under uncertainty arises when a system does not have all the needed information in its information base and a query is being posed whose answer is not directly deducible, so that it has to come up with the most appropriate (approximate) answer. This is one of the ongoing challenges in the field of expert systems (Tocatlidou, Passam, Sideridis, \& Yialouris, 2003).

On the other hand a rule-based approach is the most common and effective way of acquiring and storing knowledge in expert system. The rules thus developed can guide the user in making effective decisions. However, there are some issues still not addressed, and they are:

a. Rules may be used for making certain recommendations by the manager when new case scenarios arise. Research is required to find out how feedback from managers or experts may be used to refine the rules. This would help us to understand the robustness and tolerance of the derived rules from new case scenarios. 
b. Lack of integration of the logic rule based approach with other approaches for deriving rules in expert systems. For instance, genetic algorithms may be used to help identify variables critical to decision making before applying the logic based rule induction approach. Alternatively, a logic based rule induction approach can be applied to identify relationships between subsets of existing information, and genetic algorithms can be used to find out which of the rules would be robust in prediction and in validation (Mak \& Blanning, 2003).

\section{Future directions in case base reasoning (CBR)}

Conventional CBR has the limitation that it has no criterion for choosing the nearest cases based on the probabilistic similarity of cases. The conventional CBR technique retrieves a fixed number of neighbours in observational space. Thus, it always selects the same number of neighbours irrespective of an optimal number of similar neighbours according to target cases. This fixed number of neighbours raises a problem when some target cases should consider more similar cases while others fewer ones. In addition, the whole issue of measuring similarity between cases is problematic, especially when cases are represented as non-numeric components with complex relation between components.

Also, a problem occurs with conventional CBR when there are too many cases equally distant from target cases. Thus, it does not guarantee optimal similar neighbours for various target cases, which leads to the weakness of lowering predictability due to deviation from desired similar neighbours (Park et al., 2006).

\section{Recommendations for future asset information management systems}

If you do not know where you are heading, any road will take you there. Additionally, if you always do what you've always done, you will always get what you've always got (MRO, 2004b). Generally an Expert System for preventive maintenance operations lacks a comprehensive frame work for prioritization of preventive maintenance (Fonseca, 2000). Thus a step change in Predictive Maintenance of Assets requires a clear understanding of what an organization aspires to achieve with their assets and a radically new way of designing and implementing Asset Information Management Systems.

In line with the above observation, we propose the fusion of an Expert System with a fuzzy logic scheme that is capable of providing a better way of decision making with predictive maintenance in the Predictive Maintenance of Assets Management regime. The Expert System is used to enhance the quality of predictive maintenance through more accurate computer based decision analysis as compared to following a human centred approach. In addition, it can have the fuzzy logic reasoning ability that assists in the decision-making process.

One vital component of an asset management system is its Expert System module. We recommend the inclusion of the following distinctive features in any Expert System targeted at improving the performance of Asset management:

a. An Expert System containing semantic domain knowledge for defect identification and prioritization of maintenance activities (Nemati, Steiger, Iyer, \& Herschel, 2002).

b. A Hybrid fuzzy logic expert systems in the knowledge base as it combines a rule base with a case base reasoning module which together assist in effective and efficient problem analysis, thus building a fast and efficient Predictive Maintenance Management Expert System for asset management (Isaai \& Cassaigne, 2001).

Decision making is another component, for decision support, of asset management that does intelligent and targeted information examination. This results in better quality and high speed decision making on large volumes of information. Improved quality in decision analysis can lead to better 
recommendations. Thus we recommend that a good decision analysis should include identifying, clearly representing, and formally assessing the important aspects of a decision situation, for prescribing the recommended course of action (Clemen \& Reilly, 2001).

Predictive Maintenance proposes ways to improve the accuracy in predictions in the maintenance management regime. We recommend improving the performance of scheduling by:

a. A robust predictive scheduler which maximizes the performance, regularity, and reliability in maintenance management;

b. Reduction in work load and pressure on the experts due to better code of practice by extracting tacit knowledge (Isaai \& Cassaigne, 2001).

Our review of the relevant literature leads us to recommend that a good asset management system initially reviews various key aspects of a case, i.e., Case Based Reasoning (CBR), identifying relevant conclusions.

The expert system reviews these conclusions, each of which can fall under three categories, as follows:

1. It accepts that this conclusion is appropriate, therefore no action is required as application of knowledge is already in the system or

2. It states to the system in the form of a standardized rule why the conclusion is inappropriate and what the correct conclusion should have been, if any. This will result in refinement of knowledge already in the system or finally

3. It states to the system in the form of a rule why the conclusion is appropriate, and what it should be. This will result in addition of new knowledge to the system (Bindoff, Tenni, Peterson, Kang, \& Jackson, 2007).

\section{Conclusion}

Asset management concerns itself with systematic and optimal ways of managing assets to achieve desired outcomes in a sustainable way (Brownless, 2005). We have argued that one way to achieve this is by combining an Expert System with fuzzy logic, as together they can provide a better way to deal with Predictive Maintenance of Assets.

The quality of a decision made is directly proportional to reliability of information (Vanier, 2001). We conclude that high-quality decisions cannot be expected without high-quality information. An effective system for predictive maintenance within the framework of an asset management system should incorporate an Expert System to enhance the quality of prediction. In addition, it should have fuzzy logic reasoning ability that assists in the decision making process. The essence of the proposed system is the ability to integrate reliable information of assets with DSS to make an Expert System which will ultimately guide the asset manager by suggesting standardized rules using Neuro fuzzy logic for Predictive maintenance management of assets.

\section{References}

Bindoff, I. K., Tenni, P. C., Peterson, G. M., Kang, B. H. \& Jackson, S. L. (2007). Development of an intelligent decision support system for medication review. The Journal of Clinical Pharmacy and Therapeutics, 32, 81-88.

Brownless, G. (2005). An asset management model for UK railway safety: Literature review and discussion document. Health \& Safety Laboratory

Clarke, N. (2005). Asset management and monitoring. Retrieved February 2007 From http://www.tessella.com/wp-content/uploads/2008/06/asset_management_monitoring.pdf 
Clemen, R. T., \& Reilly, T. (2001). Making hard decisions with decision tools. Belmont, CA: Duxbury Press.

Eom, S. B., Lee, S. M., Kim, E. B., \& Somarajan, C. (1998). A survey of decision support system applications (1988-1994). The Journal of the Operational Research Society, 49(2), 109-120.

Fay, A. (2002). A fuzzy knowledge-based system for railway traffic control. The Journal of Knowledge Base System, 15, 515-528.

Fonseca, J. D. (2000). A knowledge base system for preventive maintenance. The Journal of Knowledge Engineering, Expert Systems, 17(5).

Fu, L. M. (1995). Knowledge-based neural networks. Knowledge-Based Systems: Special Issue, 8(6).

Institute of Asset Management (IAM) (2003). Asset management Part 1: Specification for the optimized management of physical infrastructure assets, PAS 55-1, V-3, BSI.

Isaai, M. T., \& Cassaigne, N. P. (2001). Predictive and reactive approaches to the train- scheduling problem: A knowledge management perspective. IEEE Transactions on Systems, 34(4).

Kyle, B. R., Vanier, D. J., Kosovac, B., \& Froese, T. M. (2000). Information needs towards service life asset management. Proceedings of the $17^{\text {th }}$ International CODATA Conference, Data and Information for the Coming Knowledge Millennium.

Lee, J. K., \& Kim, J. K. (2002). A case-based reasoning approach for building a decision model. The Journal of Knowledge Engineering, Expert Systems, 19(3).

Lounis, Z., Vanier, D. J., \& Lacasse, M. A. (1999). Decision-support system for service life asset management: The Belcam Project. Proceedings of the $8^{\text {th }}$ International Conference on Durability of Building.

Mak, B., \& Blanning, R. (2003). A logic based approach to rule induction in expert system. The Journal of Knowledge Engineering, Expert Systems, 20(3).

MRO Software Inc. (2004a). Strategic asset management in the transportation industry. Retrieved March 2007 from www.mro.com/corporate/pdf/trans wp.pdfm

MRO Software Inc. (2004b). Strategic asset management methodology. Retrieved March 2007 www.mro.com/corporate/pdf/MR391_SAMM_final.pdf

Negnevitsky, M. (2002). What is artificial intelligence? Essex. Addison Wesley Press.

Nemati, H. R., Steiger, D. M., Iyer, L. S., \& Herschel, R. T. (2002). Knowledge warehouse: An architectural integration of knowledge management, decision support, artificial intelligence and data warehousing. Decision Support Systems, 33, 143-161.

Ness, D., \& Lambie, B. (2004). Raising the performance bar: Gauging asset management improvement in a government. Proceedings of Clients Driving Innovation International Conference 2004.

Network Rails. (2005). Asset managing planning. Retrieved March 2007 from http://www.networkrail.co.uk/documents/3140_2005SummaryPlan.pdf

Park, Y. J., Kim, B-C., \& Chun, S-H. (2006). New knowledge extraction technique using probability for case-based reasoning: Application to medical diagnosis. The Journal of Knowledge Engineering, Expert Systems, 23(1).

Rippen, R. (2005). Decision support system. Retrieved April 2007 http://www.tessella.com/literature/supplements/PDF/DecisionSupportSystems.pdf

Sherwin, D. (2000). A review of overall models for maintenance management. Journal of Quality in Maintenance Engineering, 6(3), 138-164.

Tocatlidou, A., Passam, H. C., Sideridis, A. B, \& Yialouris, C. P. (2003). Reasoning under uncertainty for plant disease diagnosis. The Journal of Knowledge Engineering, Expert Systems, 19(1).

Tsang, A. H. C. (1995). Condition-based maintenance: Tools and decision making. Journal of Quality in Maintenance Engineering, 1(3), 3-17. 
Vanier, D. J. (2001). Why industry needs management tools. Journal of Computing in Civil Engineering, 15(1), 35-43.

Weidl, G., Madsen, A., \& Dahlquist, E. (2002). Condition monitoring, root cause analysis and decision making support on urgency of actions. $2^{\text {nd }}$ International Conference on Hybrid Intelligent Systems.

Wenzler, I. (March 2005), Development of an Asset Management Strategy for a Network Utility Company: Lessons from a Dynamic Business Simulation Approach, simulation \& gaming, Vol.36 No.1, 75-90.

Woodhouse, J. (2000). Finding the right mix of costs, risks and performance Retrieved April 2007 from http://www.plant-maintenance.com/articles/Costriskop.pdf

Woodhouse, J. (2001). Asset management decision making. Retrieved from http://www.plantmaintenance.com/articles/AMdecisions.pdf

Yam, R. C. M., Tse, P. W., Li, L., \& Tu, P. (2001). Intelligent predictive decision support system for condition-based maintenance. The International Journal of Advanced Manufacturing Technology, 17, 383391.

Zoeteman, A. (2001). Life cycle cost analysis for managing rail infrastructure: Concept of a decision support system for railway design and maintenance. EJTIR, 1(4), 391-413.

\section{Biographies}

Rizwan Bin Faiz has obtained his Masters in Information Technology and Computer Science. His areas of research interest include Artificial Intelligence and Software Engineering. His research area for $\mathrm{PhD}$ focuses on the development of a Predictive rail track degradation model for robust and effective maintenance. He is author of eight conference and two journal papers.

Eran Edirisinghe received his B.Sc.Eng. (Hons) degree in Electronic \& Telecommunication Engineering from Moratuwa University, Sri Lanka, in October 1994. He obtained his MSc degree in Digital Communications Systems from the Department of Electronic \& Electrical Engineering, Loughborough University in September 1996 and his PhD Degree from the Department of Computer Science, Loughborough University, in September 1999. His doctoral thesis was on Data Compression of Stereoscopic Images and Video. After a short assignment as a Postdoctoral Research Fellow at Glamorgan University, Wales, he was appointed a Lecturer in Computer Science at Loughborough University. He was promoted to a Senior Lecturer in February 2004 and received the title of Reader in Digital Imaging in July 2008. He is a member of the College of Peers of the Engineering \& Physical Sciences Research Council, UK and a member of the IASTED committee in image processing. His research interests include, image and signal processing, video coding, texture synthesis and novel mobile applications. His current research is funded by the EPSRC, DTI and industry. Dr. Edirisinghe currently supervises $12 \mathrm{PhD} / \mathrm{EngD}$ students and three RAs. 